\title{
Inconsistent handedness is linked to more successful foreign language vocabulary learning
}

\author{
VERA KEMPE \\ University of Abertay, Dundee, Scotland \\ Patricia J. BROOKS \\ College of Staten Island, City University of New York, Staten Island, New York, \\ and The Graduate Center, City University of New York, New York \\ AND \\ StePhen D. Christman \\ University of Toledo, Toledo, Ohio
}

\begin{abstract}
The study examined correlations between incidental learning of foreign words and interhemispheric connectivity, operationalized as consistency of hand preference, using pooled data of five experiments on adult foreign language learning $(N=242)$. Inconsistent hand preference was found to be positively correlated with vocabulary learning even after effects of cognitive variables (verbal working memory capacity and nonverbal IQ), identified previously as predictive of successful foreign-language vocabulary learning, were partialled out. This observed relationship between handedness consistency and vocabulary learning persisted when left-handed and right-handed individuals were analyzed separately, and there was no overall difference in performance between left- and right-handers. The findings confirm an association between degree of handedness and verbal episodic memory.
\end{abstract}

Individuals differ in their ability to learn words in a new language. One obvious source of this variability is working memory capacity, which may constrain the extent to which new phonological strings are encoded and retained. Specifically, the phonological loop, a subsystem of verbal working memory, may serve as a word-learning device controlling the storage of phonological strings (Baddeley, Gathercole, \& Papagno, 1998) via a sequence memory that encodes the serial order of phonemes, syllables, and words (Gupta, 2003). Consequently, measures of short-term verbal working memory capacity correlate with vocabulary acquisition in children (Gathercole, Hitch, Service, \& Martin, 1997) and foreign language learners (Service, 1992).

However, in natural learning situations novel words are encountered in specific semantic and referential contexts. There is good evidence that memory for newly learned vocabulary has a strong episodic component. Using evoked response potentials, Perfetti, Wlotko, and Hart (2005) demonstrated that, unlike unfamiliar words or words well established in the learner's vocabulary, recently encountered words, the meanings of which had been provided in a word-learning task, produced a late positive wave peaking at around $600 \mathrm{msec}$ after word onset, which is considered to be a marker of an episodic memory trace (Curran, 1999). This suggests that over a lifetime an individual ac- cumulates episodic memory traces representing specific experiences with words, which collectively constitute the rich knowledge base of native language vocabulary (Perfetti \& Hart, 2002; Reichle \& Perfetti, 2003). Indeed, amnesic individuals who have lost their ability to form episodic memories tend to be unsuccessful at learning new vocabulary (Temple \& Richardson, 2006).

Recent evidence shows asymmetrical involvement of the cortical hemispheres in episodic memory tasks. The hemispheric encoding/retrieval asymmetry model was developed to account for greater activation in the left prefrontal cortex during encoding and greater activation in the right prefrontal cortex during recall or recognition (Tulving, Kapur, Craik, Moscovitch, \& Houle, 1994) ${ }^{1}$. The model predicts that interhemispheric connectivity modulates performance in episodic memory tasks; indeed, splitbrain patients demonstrate deficits in episodic but not in semantic memory (Cronin-Golomb, Gabrieli, \& Keane, 1996). One way to operationalize degree of interhemispheric connectivity is to utilize individual differences in consistency of hand preference; 2 this is assumed to be negatively related to interhemispheric connectivity since the size of the corpus callosum is significantly smaller in consistent right-handers (Witelson, 1989). Inconsistently handed individuals are advantaged in a variety of episodic memory tasks, including earlier offset of childhood am-

V. Kempe, v.kempe@abertay.ac.uk 
nesia (Christman, Propper, \& Brown, 2006), better source memory (Christman, Propper, \& Dion, 2004; Lyle, McCabe, \& Roediger, 2008), and better episodic recall of laboratory and real-world memories (Lyle, Logan, \& Roediger, 2008; Propper, Christman, \& Phaneuf, 2005).

If enhanced interhemispheric connectivity facilitates episodic memory recall, inconsistently handed individuals should show an advantage in learning new vocabulary after variance associated with cognitive variables, such as working memory capacity and nonverbal intelligence, has been removed. Nonverbal intelligence may free attentional resources for incidental vocabulary learning by facilitating learning of structural properties of a new language (Kempe \& Brooks, 2008). To examine this prediction, we present pooled data from five foreign-language-learning studies in which adults were given associations of short Russian phrases and their meanings in a variety of comprehension and production tasks, thus mimicking vocabulary learning as it may occur in naturalistic situations.

\section{METHOD}

\section{Participants}

Seventy men and 170 women ( $M=22$ years, $S D=6.2$; age range, $17-55$ years) participated in five Russian-grammar-learning experiments. All were native speakers of American English with no prior knowledge of Russian. Eighty-one women and 35 men participated in two gender-learning experiments; 90 women and 37 men participated in three case-learning experiments.

\section{Materials, Procedure, and Scoring}

In all five experiments, participants were shown line drawings of common objects (e.g., a red fence) and heard corresponding Russian phrases (e.g., krasnyj zabor), which they had to repeat twice. The Russian phrases were then presented with pairs of drawings (a target and a distractor), and participants had to select the corresponding drawing for each phrase. Finally, they were instructed to produce inflected forms of the Russian words or to categorize them according to grammatical category. In one gender-learning experiment, participants saw pictures of colored objects and had to produce color adjectives, which were inflected to agree with the gender of the noun [e.g., krasnyj zabor (red MASCULINE; fence MASCULINE/NOMINATIVE) or krasnaya skripka (red FEMININE; violin FEMININE/NOMINATIVE)]. In a second gender-learning experiment, participants simply heard the nouns and were instructed to categorize them according to gender (see Kempe, Brooks, \& Kharkhurin, 2009). In three case-learning experiments, participants saw drawings of a protagonist moving toward or away from (or, alternatively, positioned above or below) the object and had to produce Russian prepositional phrases describing the protagonist's movement, for example, $k$ zaboru (toward the fence, MASCULINE/DATIVE), using inflections marking the morphological case of the noun (genitive and dative in two experiments, instrumental and locative in the third) (Brooks, Kempe, \& Sionov, 2006; Kempe \& Brooks, 2008). These tasks were presented during the training sessions, which were administered on different days within a 2-week period. The gender-learning experiments each contained 30 nouns repeated over four training sessions; the case-learning experiments each contained 24 nouns repeated over six training sessions. At the end of training, participants were tested on their mastery of gender-marking or case-marking inflections, using familiar and novel nouns.

\section{Vocabulary Learning}

At the end of the final session, participants were prompted to recall the Russian nouns corresponding to the depicted objects. As the language-learning tasks focused on inflectional morphology, the vocabulary recall test was unexpected and designed to tap into participants' memory of the word forms accompanying each picture. Participants were shown drawings of each object and were instructed to say their Russian names, even encouraged to guess. If they could not recall the Russian name of the object, they were instructed to say "I don't know." Responses were audiotaped.

Vocabulary test scores were obtained by counting the number of correctly recalled nouns. Since participants often used inflected forms of nouns, an item was scored as correct if the response started with the correct phoneme and if the root resembled the correct word, regardless of noun ending produced (i.e., errors in case-marking inflections were not scored as vocabulary recall errors). For example, the forms zabor, zabora, and zaboru, as well as the incorrect forms zabore and zabory, were all counted as correct recalls. The rationale behind this scoring principle was to focus on recall of the lexical root and to not penalize participants for incorrect inflections.

Handedness was assessed using the Edinburgh Handedness Inventory (EHI; Oldfield, 1971), which asks participants to rate how often they carry out 10 manual actions (e.g., striking a match, opening a jar) with the right or left hand. Scores are assigned on a scale from -100 (extreme left-handedness) to 100 (extreme right-handedness). Handedness consistency was determined using the absolute values of the EHI scores. This measure disregards the direction of handedness (left or right) and focuses on the consistency of lateralization of hand preference, with higher values indicating more consistent handedness. Verbal working memory was assessed with a reading span task, using materials and procedures outlined in Daneman and Carpenter (1980). Participants were asked to read aloud a set of unrelated sentences and to recall the final word of each sentence in the set. Scoring involved counting the number of correctly reproduced words out of the total of 70. Finally, nonverbal intelligence was assessed using the Cattell Culture-Fair Test of Nonverbal Intelligence, Scale 3, Form A (Cattell \& Cattell, 1973), containing four sets of multiple-choice problems to be completed within the time allotted by the test manual. Order of administration of these tests was counterbalanced across sessions.

\section{RESULTS}

Table 1 presents the percentage of correctly recalled Russian nouns in the five experiments. The differences in vocabulary recall across experiments simply reflect the fact that the experiments contained different nouns, different numbers of nouns, and different numbers of training sessions, as detailed in the Method section. The last column presents partial correlations between handedness consistency and vocabulary recall, controlling for effects of culture-fair IQ and reading span, which assess the independent effect of handedness consistency. The

Table 1

Mean Percentage of Correctly Recalled Russian Nouns in the Five Russian Grammar Learning Experiments

\begin{tabular}{lcccc}
\hline Experimental Study & \% Correct & $S D$ & $N$ & Partial $r$ \\
\hline Gender Learning & & & & \\
Experiment 1 & 37.7 & 14.5 & 49 & $-.33^{*}$ \\
Experiment 2 & 23.9 & 12.7 & 64 & .02 n.s. \\
Case Learning & & & & \\
Experiment 1 & 79.5 & 13.8 & 56 & $-.40^{* *}$ \\
Experiment 2 & 74.2 & 29.7 & 53 & $-.24^{\dagger}$ \\
Experiment 3 & 65.2 & 20.3 & 20 & $-.23^{\dagger}$ \\
\hline
\end{tabular}

Note-The last column lists partial correlations of handedness consistency with correctly recalled nouns after culture-fair IQ and reading span have been partialled out. $\quad{ }^{*} p<.05 .{ }^{* *} p<.01 . \quad{ }^{\dagger} p=.09 . \quad{ }^{\dagger} p=.37$. 
negative correlation between handedness consistency and vocabulary recall was significant in two experiments, fell short of significance in one, and failed to reach significance, presumably due to a lack of statistical power, in the case-marking experiment with the smallest number of participants. Only in one out of five experiments (gender-learning Experiment 2) was there no correlation and no trend. This was the experiment with the lowest performance in vocabulary recall, where a floor effect may have obscured the expected negative correlation. Because of the high degree of similarity in procedures and materials across the five experiments, we deemed it justified to pool the data to gain statistical power. To account for differences in the target nouns and the numbers of nouns and training sessions across experiments, we computed $z$ scores for vocabulary recall for each experiment separately. The partial correlation between handedness consistency and vocabulary $z$ scores, controlling for effects of culture-fair IQ and reading span, was $r(238)=$ $-.23, p<.001$, confirming that in the pooled sample, inconsistent hand preference was reliably associated with better vocabulary recall.

Table 2 shows the correlations between culture-fair IQ, reading span, and vocabulary $z$ scores for the entire sample. As expected, culture-fair IQ and reading span were positively correlated with each other, presumably reflecting a shared executive component. The correlations with vocabulary recall suggest that both nonverbal intelligence and verbal working-memory capacity play a role in the retrieval of novel verbal materials.

When culture-fair IQ and reading span were entered at the first step into a stepwise regression analysis with vocabulary $z$ scores as dependent variable, they jointly accounted for $14.4 \%$ of variance $[F(2,239)=20.1, p<$ $.001]$. Both of these cognitive tasks were positively related to vocabulary recall (culture-fair IQ, $\beta=24$; reading span, $\beta=.22$ ). When handedness consistency was entered at the second step, it accounted for an additional $4.5 \%$ of variance [partial $F(1,238)=13.3, p<.001]$. Auxiliary regression analyses confirmed that multicollinearity did not affect the stability of regression coefficients (all $R^{2} \mathrm{~s}<$ .08 , variance inflation factors $<1.1$ ).

Given the relatively large sample size, we had the opportunity to explore whether the relationship between handedness consistency and vocabulary recall held equally for men and women; this is important, given that neuroanatomical findings imply potential gender differences in interhemispheric connectivity (Habib et al., 1991; Witelson, 1989; Witelson \& Goldsmith; 1991). Note that men and women did not differ in overall vocabulary recall; mean

Table 2

Correlation Between Culture-Fair IQ, Reading Span and Vocabulary Recall $z$ Scores

\begin{tabular}{lcc}
\hline & Reading Span & Vocabulary \\
\hline Culture fair & $.27^{* * *}$ & $.32^{* * *}$ \\
Reading span & & $.28^{* * *}$ \\
${ }^{* * * *} p<.001$. &
\end{tabular}

Table 3

Partial $R^{2}$ and $\beta$ Values Obtained in Stepwise Regression Analyses of Culture-Fair (CF) IQ, Reading Span (Entered at Step 1), and Handedness Consistency (Entered at Step 2) Onto Vocabulary $z$ Scores for Women and Men Separately

\begin{tabular}{|c|c|c|c|c|}
\hline & \multicolumn{2}{|c|}{ Women $(n=170)$} & \multicolumn{2}{|c|}{$\operatorname{Men}(n=72)$} \\
\hline & Partial $R^{2}$ & $\beta$ & Partial $R^{2}$ & $\beta$ \\
\hline \multicolumn{5}{|l|}{ Step 1} \\
\hline $\begin{array}{l}\text { CF IQ and } \\
\text { reading span }\end{array}$ & $.115^{* * *}$ & $\begin{array}{l}.23^{* *} \\
.19^{*}\end{array}$ & $.209^{* * *}$ & $\begin{array}{l}.32^{* *} \\
.27^{*}\end{array}$ \\
\hline \multicolumn{5}{|l|}{ Step 2} \\
\hline Handedness consistency & $.026^{*}$ & $-.16^{*}$ & $.094^{* *}$ & $-.32^{* *}$ \\
\hline
\end{tabular}

vocabulary $z$ scores were 0.09 for men and -0.04 for women $[t(240)=0.96, p=.3]$. Table 3 shows the results of the same stepwise regression analysis for both genders separately. Whereas the independent effect of handedness consistency was significant for both men and women, it accounted for an additional $9.4 \%$ in men but for only $2.5 \%$ in women. To test whether this difference was significant, we performed another stepwise regression on the entire sample, this time entering culture-fair IQ and reading span at the first step, handedness consistency at the second step, and the interaction between gender and handedness consistency at the third step. This analysis confirmed the previous results, but did not show a significant effect of the interaction between gender and handedness consistency $(p=.7)$. This demonstrates an independent effect of handedness consistency on vocabulary recall in both men and women.

Finally, we examined whether the traditional classification of left- versus right-handedness would be equally predictive of successful vocabulary recall, by comparison with a measure of handedness consistency. Left-handers were defined as participants with an EHI score of 0 or below. There were no group differences in vocabulary recall between left- and right-handers; the mean vocabulary $z$ scores were 0 for both left- and right-handers $[t(240)=0, p=1]$. Table 4 presents the results of the stepwise regression performed for left- and right-handers separately. Despite having only 23 left-handers in our sample, we found a significant effect of handedness consistency in both groups, suggesting

Table 4

Partial $R^{2}$ and $\beta$ Values Obtained in Stepwise Regression Analyses of Culture-Fair (CF) IQ, Reading Span (Entered at Step 1), and Handedness Consistency (Entered at Step 2) Onto Vocabulary $z$ Scores for RightHanders and Left-Handers Separately

\begin{tabular}{|c|c|c|c|c|}
\hline & \multicolumn{2}{|c|}{$\begin{array}{l}\text { Right-Handers } \\
\quad(n=219)\end{array}$} & \multicolumn{2}{|c|}{$\begin{array}{l}\text { Left-Handers } \\
\quad(n=23)\end{array}$} \\
\hline & Partial $R^{2}$ & $\beta$ & Partial $R^{2}$ & $\beta$ \\
\hline \multicolumn{5}{|l|}{ Step 1} \\
\hline $\begin{array}{l}\text { CF IQ and } \\
\text { reading span }\end{array}$ & $.165^{* * *}$ & $\begin{array}{l}.28^{* * *} \\
.23^{* *}\end{array}$ & .017 & $\begin{array}{l}.08 \\
.08\end{array}$ \\
\hline \multicolumn{5}{|l|}{ Step 2} \\
\hline Handedness consistency & $.034^{* *}$ & $-.19^{* *}$ & $.197^{*}$ & -.46 \\
\hline
\end{tabular}


that this effect was independent of the traditional classification of left- versus right-handedness. Again, the size of the effect of handedness consistency was different in the two groups: $3.4 \%$ of variance accounted for in right-handers and $19.7 \%$ in left-handers. A stepwise regression on the entire sample with the interaction between handedness consistency and handedness entered at the last step showed that this interaction was not significant $(p=.1)$, suggesting that the difference in effect sizes between left- and right-handers was not reliable.

\section{DISCUSSION}

Our findings demonstrate a clear negative relationship between handedness consistency and vocabulary learning: Adults who reported inconsistent hand preference showed an advantage in vocabulary learning over those who reported consistent hand preference. Since handedness consistency is taken as an indicator of interhemispheric connectivity, our findings suggest that greater interhemispheric activation is linked to better performance in vocabulary learning, over and above the effects of the cognitive factors of verbal working memory capacity and nonverbal intelligence. This link between degree of hand preference and vocabulary recall, although relatively small in size, appears to be independent of direction of handedness and participant gender. It remains to be determined whether this effect arises during vocabulary encoding or retrieval.

Christman et al. (2006) have suggested that handedness consistency effects emerge during memory retrieval. They demonstrated that individuals with consistent handedness preference reported later offset of childhood amnesia. Moreover, when consistently handed individuals engaged in bilateral eye movements, which have been shown to enhance episodic retrieval (Christman, Garvey, Propper, $\&$ Phaneuf, 2003), they reported memories from about the same age as did individuals with inconsistent handedness preference in the absence of eye movements. However, the results reported here do not exclude the possibility that handedness differences may also reflect differences in encoding. For example, Ranganath, Johnson, and D'Esposito (2003) have shown that the same bilateral prefrontal regions are active during encoding and recognition in long-term memory as well as encoding into working memory, suggesting that greater interhemispheric connectivity might also benefit the integration of information during encoding.

A possible candidate for the origin of the effect of handedness consistency is the "episodic buffer" (Baddeley, 2000), a component of working memory that serves to integrate information in working memory with different sources of information in long-term episodic memory. For example, during the first session in our experiments, participants had to link phonological loop representations of newly presented second-language words with preexisting long-term memory representations of the associated objects. In subsequent sessions, they had to integrate the phonological loop representations with earlier phono- logical representations as well as with word meanings. Recent evidence suggests that episodic buffer activity is associated with bilateral patterns of cortical activation (e.g., Kiss, Watter, Heisz, \& Shedden, 2007; Zhang et al., 2004), consistent with the current idea that increased interhemispheric connectivity allows for better integration of left- and right-hemispheric processing.

An episodic benefit associated with greater interhemispheric connectivity is also consistent with the lexical quality hypothesis (Perfetti \& Hart, 2002; Reichle \& Perfetti, 2003), which suggests that the size and structural organization of one's word knowledge base determines how episodic traces for new words can be integrated into memory. This is in line with Perfetti et al.'s (2005) view that vocabulary learning involves functional encoding of word episodes. Thus, the observed effect of handedness consistency on foreign vocabulary learning is compatible with the idea that greater interhemispheric activation may facilitate both encoding and subsequent retrieval of such episodic traces.

It is interesting to note that in our data, handedness consistency was linked to vocabulary recall in men and in women. Several analyses of corpus callosum volume have reported significant associations between consistency of hand preference and callosum size for men, but not for women (Witelson, 1989; Witelson \& Goldsmith; 1991), and there is evidence that the posterior end of the corpus callosum - that is, the splenium - is larger and more bulbous in women than in men (Habib et al., 1991). Despite these suggestions of gender differences in anatomical features of interhemispheric connectivity, we did not find differences between the genders in the effect of handedness consistency on the learning of foreign words. This, of course, does not disprove the hypothesis that there may be gender differences in interhemispheric connectivity, or in the impact of interhemispheric connectivity on cognitive functions. Given the size of our sample, our finding suggests that the behavioral manifestations of such gender differences, if they exist at all, may be quite small. Perhaps handedness consistency, as measured by the EHI, is just too coarse a measure to be sensitive to more subtle gender differences in interhemispheric connectivity. Note that the lack of gender effects reported here does not call into question the existence of gender differences in functional specialization of the hemispheres for which there is considerable empirical evidence (e.g., Ragland, Coleman, Gur, Glahn, \& Gur, 2000; Shaywitz et al., 1995).

In general, our findings point to the importance of examining the role of callosally mediated access to righthemispheric functioning in first and second language acquisition. Future studies in this domain should include handedness consistency as a variable so that variability associated with individual differences in interhemispheric connectivity becomes part of the effect term rather than the error term. In addition, as greater interhemispheric connectivity in individuals with inconsistent hand preference provides them with better access to the right hemisphere (Christman, Henning, Geers, Propper, \& Niebauer, 2008), and the right hemisphere has been shown to play a role in language and vocabulary acquisition (Bates et al., 
1997; Ince \& Christman, 2002), examining handedness preferences may serve as a tool for further investigating the role of the right hemisphere in language learning.

\section{AUTHOR NOTE}

A portion of this article was presented at the 47th Annual Meeting of the Psychonomic Society, and was supported by a grant to P. J. Brooks from Language Learning: A Journal of Research in Language Studies. We thank Ariel Sionov and Anatoliy Kharkhurin for their assistance with data collection, and Keith Lyle for helpful comments on an earlier draft of the article. Correspondence should be addressed to V. Kempe, Psychology (SHS), University of Abertay, Room Level 5, Kydd Building, Bell Street, Dundee DD1 LHG, Scotland (e-mail: v.kempe@abertay.ac.uk).

\section{REFERENCES}

Babiloni, C., Vecchio, F., Cappa, S., Pasqualetti, P., Rossi, S., Miniussi, C., \& Rossini, P. M. (2006). Functional frontoparietal connectivity during encoding and retrieval processes follows HERA model: A high-resolution study. Brain Research Bulletin, 68, 203-212. doi:10.1016/j.brainresbull.2005.04.019

BADDELEY, A. D. (2000). The episodic buffer: A new component of working memory? Trends in Cognitive Sciences, 4, 417-423. doi:10.1016/ S1364-6613(00)01538-2

Baddeley, A. D., Gathercole, S. E., \& Papagno, C. (1998). The phonological loop as a language learning device. Psychological Review, 105, 158-173. doi:10.1037/0033-295X.105.1.158

Bates, E., Thal, D., Trauner, D., Fenson, J. Aram, D., Eisele, J., \& NAss, R., (1997). From first words to grammar in children with focal brain injury. Developmental Neuropsychology, 13, 275-343.

Brooks, P. J., Kempe, V., \& Sionov, A. (2006). The role of learner and input variables in learning inflectional morphology. Applied Psycholinguistics. 27, 185-209. doi:10.1017/S0142716406060243

Cattell, R. B., \& Cattell, H. E. P. (1973). Measuring intelligence with the culture-fair tests. Champaign, IL: Institute for Personality and Ability Testing.

Christman, S. D., Garvey, K. J., Propper, R. E., \& Phaneuf, K. A. (2003). Bilateral eye movements enhance the retrieval of episodic memories. Neuropsychology, 17, 221-229. doi:10.1037/0894 $-4105.17 .2 .221$

Christman, S. D., Henning, B., Geers, A. L., Propper, R. E., \& Niebauer, C. L. (2008). Mixed-handed persons are more easily persuaded and are more gullible: Interhemispheric interaction and belief updating. Laterality, 13, 403-426. doi:10.1080/13576500802079646

Christman, S. D., Propper, R. E., \& Brown, T. J. (2006). Increased interhemispheric interaction is associated with earlier offset of childhood amnesia. Neuropsychology, 20, 336-345. doi:10.1037/0894 $-4105.20 .3 .336$

Christman, S. D., Propper, R. E., \& Dion, A. (2004). Increased interhemispheric interaction is associated with decreased false memories in a verbal converging semantic associates paradigm. Brain \& Cognition, 56, 313-319. doi:10.1016/j.bandc.2004.08.005

Cronin-Golomb, A., Gabrieli, J. D. E., \& Keane, M. M. (1996). Implicit and explicit memory retrieval within and across the disconnected cerebral hemispheres. Neuropsychology, 10, 254-262. doi:10.1037/0894-4105.10.2.254

Curran, T. (1999). The electrophysiology of incidental and intentional retrieval: ERP old/new effects in lexical decision and recognition memory. Neuropsychologica, 37, 771-785.

Daneman, M., \& CARPenter, P. A. (1980). Individual differences in working memory and reading. Journal of Verbal Learning \& Verbal Behavior, 19, 450- 466.

Gathercole, S. E., Hitch, G. J., Service, E., \& Martin, A. J. (1997). Short-term memory and new word learning in children. Developmental Psychology, 33, 966-979. doi:10.1037/0012-1649.33.6.966

GupTA, P. (2003). Examining the relationship between word learning, nonword repetition, and immediate serial recall in adults. Quarterly Journal of Experimental Psychology, 56A, 1213-1236. doi:10.1080/02724980343000071

Habib, M., Gayraud, D., Oliva, A., Regis, J., Salamon, G., \& KhaliL, R. (1991). Effects of handedness and sex on the morphol- ogy of the corpus callosum: A study with brain magnetic resonance imaging. Brain \& Cognition, 16, 41-61. doi:10.1016/0278 -2626(91)90084-L

Habib, R., Nyberg, L., \& Tulving, E. (2003). Hemispheric asymmetries of memory: The HERA model revisited. Trends in Cognitive Sciences, 7, 241-245. doi: 10.1016/S1364-6613(03)00110-4

InCE, E., \& Christman, S. D. (2002). Semantic representation of word meanings by the cerebral hemispheres. Brain \& Language, 80, 393420. doi:10.1006/brln.2001.2599

Kempe, V., \& Brooks, P. J. (2008). Second language learning of complex inflectional systems. Language Learning, 58, 703-746.

Kempe, V., Brooks, P. J., \& Kharkhurin, A. (2009). Cognitive predictors of Russian gender categorization in L2-learners. Manuscript submitted for publication.

Kiss, I., Watter, S., Heisz, J. J., \& Shedden, J. M. (2007). Control processes in verbal working memory: An event-related potential study. Brain Research, 1172, 67-81. doi:10.1016/j.brainres.2007.06.083

Lyle, K. B., Logan, J., \& Roediger, H. L., III (2008). Eye movements enhance memory for individuals who are strongly right-handed and harm it for individuals who are not. Psychonomic Bulletin \& Review, 3, 515-520.

Lyle, K. B., McCabe, D. P., \& Roediger, H. L., III (2008). Handedness is related to memory via interhemispheric interaction: Evidence from paired associate recall and source memory tasks. Neuropsychology, 22, 523-530. doi:10.1037/0894-4105.22.4.523

McDermott, K. B., Petersen, S. E., Bruckner, R. L., Kelley, W. M., \& SANDERS, A. L. (1999). Set- and code-specific activation in the frontal cortex: An fMRI study of encoding and retrieval of faces and words. Journal of Cognitive Neuroscience, 11, 631-640.

OLDFIELD, R. (1971). The assessment and analysis of handedness: The Edinburgh Inventory. Neuropsychologica, 9, 97-113. doi:10.1016/0028 -3932(71)90067-4

Perfetti, C. A., \& Hart, L. (2002). The lexical quality hypothesis. In L. Verhoeven, C. Elbro, \& P. Reitsma (Eds.), Precursors of functional literacy (pp. 189-213). Amsterdam/Philadelphia: John Benjamins.

Perfetti, C. A., Wlotko, E. W., \& Hart, L. A. (2005). Word learning and individual differences in word learning reflected in event-related potentials. Journal of Experimental Psychology: Learning, Memory, \& Cognition, 31, 1281-1292. doi:10.1037/0278-7393.31.6.1281

Propper, R. E., Christman, S. D., \& Phaneuf, K. A. (2005). A mixedhanded advantage in episodic memory: A possible role of interhemispheric interaction. Memory \& Cognition, 33, 751-757.

Ragland, J. D., Coleman, A. R., Gur, R. C., Glahn, D. C., \& Gur, R. E. (2000). Sex differences in brain-behavior relationships between verbal episodic memory and resting regional cerebral blood flow. Neuropsychologica, 38, 451-461.

Ranganath, C., Johnson, M., \& D’Esposito, M. (2003) Prefrontal activity associated with working memory and episodic longterm memory. Neuropsychologia, 41, 378-389. doi:10.1016/S0028 -3932(99)00086-X

Reichle, E. D., \& Perfetti, C. A. (2003). Morphology in word identification: A word-experience model that accounts for morpheme frequency effects. Scientific Studies of Reading, 7, 219-237. doi:10.1207/ S1532799XSSR0703_2

Service, E. (1992). Phonology, working memory, and foreign-language learning. Quarterly Journal of Experimental Psychology, 45A, 2150 .

Shaywitz, B. A., Shaywitz, S. E., Pugh, K. R., Constable, R. T., Skudlarski, P., Fulbright, R. K., Et Al. (1995). Sex differences in the functional organization of the brain for language. Nature, $\mathbf{3 7 3}$, 607-609. doi:10.1038/373607a0

Temple, C. M., \& Richardson, P. (2006). Developmental amnesia: Fractionation of developing memory systems. Cognitive Neuropsychology, 23, 762-788. doi:10.1080/02643290500538315

Tulving, E., Kapur, S., Craik, F. I. M., Moscovitch, M., \& Houle, S. (1994). Hemispheric encoding/retrieval asymmetry in episodic memory: Positron emission tomography findings. Proceedings from the National Academy of Sciences, 91, 2016-2020.

Witelson, S. F. (1989). Hand and sex differences in the isthmus and genu of the human corpus callosum. Brain, 112, 799-835.

Witelson, S. F., \& GoldSMith, C. H. (1991). The relationship of hand preference to the anatomy of the corpus callosum in men. Brain Research, 545, 175-182. 
Zhang, D., Zhang, X., Sun, X., Li, Z., Wang, Z., He, S., \& Hu, X. (2004). Cross-modal temporal order memory for auditory digits and visual locations: An fMRI study. Human Brain Mapping, 22, 280289. doi: $10.1002 / \mathrm{hbm} .20036$

\section{NOTES}

1. This asymmetry is orthogonal to asymmetries associated with the processing of verbal versus spatial materials, suggesting that component- specific hemispheric specialization in episodic memory may be independent of content in some parts of the prefrontal cortex (Babiloni et al., 2006; Habib, Nyberg, \& Tulving, 2003; McDermott, Petersen, Brackner, Kelley, \& Sanders, 1999).

2 . The terms consistent versus inconsistent are compatible with the terms strong versus weak used in some of the handedness literature.

(Manuscript received November 20, 2008; revision accepted for publication February 17, 2009.) 